MILITARY TECHNICAL COLLEGE CAIRO - EGYPT

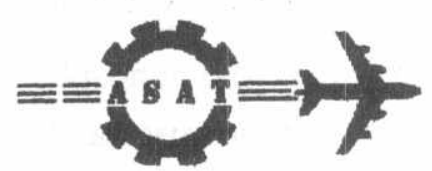

$8^{\text {th }}$ INTERNATIONAL. CONF. ON AEROSPACE SCIENCES \& AVIATION TECHNOL_OGY

\title{
Orbit and Clock Error Estimation for ARWAAS
}

\author{
Sameeh.Y. Issa, Matar. A. Matar, and H.M.S.Abdel-Wahab. \\ Military Technical College \\ Avionics Department
}

\begin{abstract}

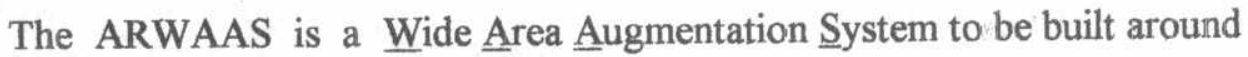
GPS constellation for improving the accuracy, integrity, and availability of the basic GPS signals over the ARab Countries region This system should allow GPS to be used as a primary means of navigation for en-route travel and for Category I approaches to selected airports throughout the region. This system would be extended to cover the Middle East region as well as most of the African continent. The system inherently should correct for ephemeris error (through satellite orbit estimation) and some other sources of errors.

In this paper, several techniques for GPS satellite orbit estimation (Non-dynamic, Kinematic, and Dynamic) for ephemeris error calculation are considered. Moreover, the problem of clock error estimation and reduction is investigated. A Least-Square algorithm is developed for predicting the system performance under an initial layout for the ARWAAS so as to evaluate the anticipated performance.
\end{abstract}

\section{Introduction}

The Wide Area Differential GPS (WADGPS) or Wide Area Augmentation System (WAAS) is being developed to mitigate some of the GPS problems through additional ranging and correction signals generated by supporting ground network and broadcast by geo-stationary satellites. The WAAS network includes at least one Wide area Master Station (WMS), a number of Wide area Reference Stations (WRS)or monitor stations, and communication links. The layout of the ARWAAS system [1] is depicted in Fig.1.

The WRS station measure the pseudo-ranges to all the satellite in view, the ionospheric delay characteristics if possible, then it remove the ionosphere and the troposphere delay estimates from the carrier smoothed pseudorange measurements. Also removed are the satellite clock corrections and the actual range from the WRS to the satellite. The resulting residuals include satellite ephemeris error, satellite clock error, receiver clock error and measurement noise. The WRS sends those residuals, along with their confidence limits to the master station. 
The master station collect the measurements from all the monitor stations and compute the GPS satellite position correction, and the ionospheric parameters, then broadcast this correction to the user through a geo-stationary satellite. Being the heart of this system (ARWAAS), we concentrate upon the processing algorithms and functions required and implemented for the system in the WMS station[2].

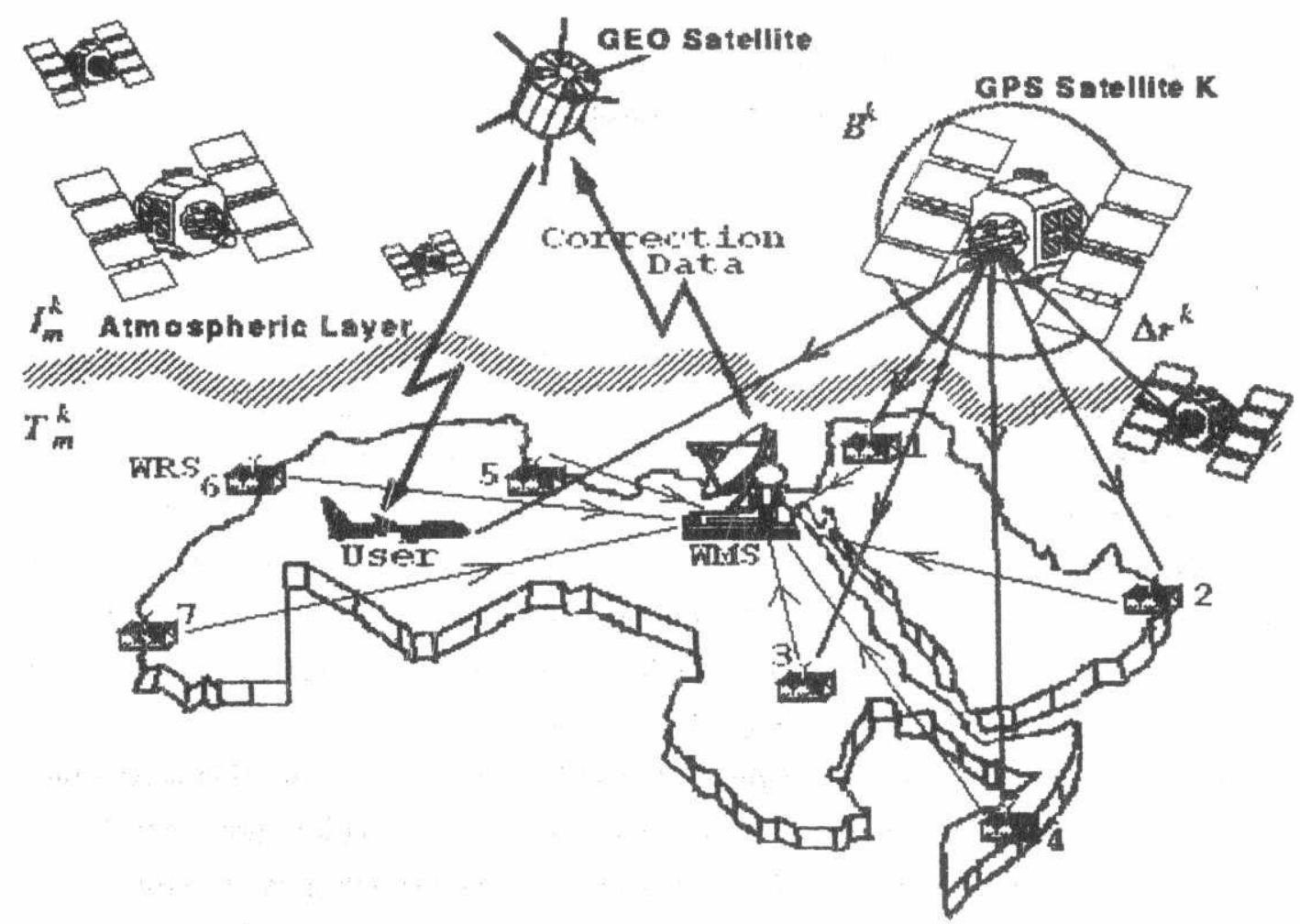

Fig (1) The layout of ARWAAS system.

The WM.S task comprises two main estimators:

- The orbit and clock error estimator. It uses the iono- and tropo-free pseudorange residuals from the set of WRS to estimate the clock and location errors for each satellite in view of the network. This is the core of the present paper.

- The ionospheric delay estimator. This estimator uses the ionospheric delay data to generate a grid of locations that uniformly covers the service area.

In this paper we, the master station processing techniques relevant to the clock error estimation are dis,cussed in Section 2 for the GPS satellite. Section 3 considers the various approaches for satellite ephemeris error calculation while section 4 presents a Least-square Method for the satellite ephemeris error calculation on the basis of the non-dynamic approach for the AP.WAAS. Analysis of results leads to the conclusions given in section 5 . 


\section{The Clock Error Estimation Techniques:}

At the WRS site, the pseudorange residuals include satellite ephemeris error, satellite clock error, receiver clock error and measurement noise. These residuals, along with their confidence limits are sent to the master station for further processing.

These smoothed pseudorange residuals are [4] modeled by the following equation:

$$
\Delta \rho_{i}^{j}=\Delta r^{j} \cdot 1_{i}^{j}+\Delta b_{i}-\Delta B^{j}+v_{i}^{j}
$$

where

$\Delta \rho_{i}^{j} \quad$ is the smoothed pseudorange residual.

$\Delta b_{i} \quad$ is the receiver clock error of the $i$-th WRS.

$\angle B^{j}$ is the clock error of the $j$-th satellite

$\angle \mathrm{r}^{\mathrm{j}}$ is the ephemeris error of the $\mathrm{j}$-th satellite

It is required to estimate both the ephemeris error and the clock error of the $j$-th satellite. The above equation engages the receiver clock error of the WRS stations as well as the measurement noise. The presence of the WRS clock error in this equation will couple the residuals: of each WRS and will result in larger dimension of corresponding state vector Hence, this nuisance parameter $\Delta b_{i}$ should be eliminated and is feasible through common-view time transfer technique.

\subsection{Common-View Time Transfer}

For the satellites in the common view of WMS and WRS stations, the difference between the $i^{\text {th }}$ receiver's clock bias and the clock bias of the master receiver is obtained through differencing the pseudorange residuals from WMS and WRS sites as follows:

$$
\begin{aligned}
& \Delta_{i, M}^{j}=\Delta \rho_{i}^{j}-\Delta \rho_{M}^{j} \\
& \Delta_{i, M}^{j}=\Delta r j\left(1_{i}^{j}-1_{M}^{j}\right)+\Delta b_{i, M}+v_{i, M}^{j}
\end{aligned}
$$

If the line of sight difference is small and/or the ephemeris error is small, a good estimate of the clock difference can be achieved by averaging the clock differences computed from all of the satellites jointly observed by both stations as follows:

$$
\Delta \hat{b}_{i, M}=\frac{1}{K_{i, M}} \cdot \sum_{j=1}^{K_{t, M}} \Delta_{i, M}^{j}
$$

where $\mathbf{K}_{\mathrm{i}, \mathrm{M}}$ is the number of satellites in the common view of both stations.

Using such multiple common satellites also reduces the error introduced by measurement noise and by ephemeris errors. In addition, this estimate is averaged over time to take advantage of the well behaved drift characteristics of the rubidium clocks at the used set of WRS stations. 
The estimate of receiver clock bias (transferred at the WMS) is then used to eliminate the term $\Delta b_{i} \quad$ in Eq (1) and all of the measurements are now in terms of a common clock, hence a "synchronized "pseudorange residuals are available and described by:

$$
\begin{aligned}
& \Delta \tilde{\rho}_{i, M}^{j}=\Delta \rho_{i}^{j}-\Delta \hat{b}_{i, M} \\
& \Delta \tilde{\rho}_{i, M}^{j}=\Delta r{ }^{j} I_{i}^{j}+\Delta b_{M}-\Delta B^{j}+v_{i}^{j}
\end{aligned}
$$

This common view synchronization results in decoupling the measurements at the various WRS. It is possible for the WMS to contain one small estimator for each of the satellites in view of the network. In contrast, if the residuals were not synchronized, then all of the observations are coupled trogether and a single large estimator must be used to solve for tens of unknowns and a complex algorithms required in such situation.

The obtained synchronized pseudorange residuals will be used as the fundamental input to the satellite ephemeris and clock estimation algorithms described below.

\section{The Orbit 'Determination / Estimation Techniques:}

Three general Techniques: Nondynamic, Kinematic and Dynamic are possible for solving 'his problem. They will outlined in the following sub-sections.

\subsection{The Nondynamic Techniques:}

This technique is based upon inverted form of the navigation fix solution yields the instarataneous position of the satellite, without the use of dynamical information. It doesn't enable to separate the ephemeris and clock errors, as does the dynamic technique. The solution is found at the WMS directly from the instantaneous measurements of the satellite position by at least 3 WRS stations [3]. This technique will be discussed and used in section 4 on the basis of a Least-Squares algorithm.

\subsection{The Kinematic Algorithms}

This technique comprises the ephemeris and ephemeris rate errors as well as clock and clock rate errors. Several approaches could be used to estimate the ephemeris errors as well as clock errors separately or jointly (simultaneously).

For separate estimation of satellite ephemeris and clock errors, a minimum norm algorithm is given in [4] where a Kinematic model for ephemeris error and a second order Gauss-Markov process model for clock error are used. This method uses single differences of synchronized pseudorange residuals, first to obtain measurement equations for ephemeris only, then to carry out the Kalman filter estimate for ephemeris and ephemeris velocity errors. After obtaining the ephemeris error estirnate, we substitute it back into Equation (4) to obtain the satellite clock error for further processing by a Kalman filter to best estimate the clock error. 
For simultaneous estimation of satellite ephemeris and clock errors, 4D Kalman filter casting is used with the state vector containing the ephemeris and ephemeris velocity errors as well as clock and clock rate errors. The following equations for the process and measurements models are used:

$$
\begin{aligned}
& X(k+1)=\Phi(k) X(k)+B(k) W(k) \\
& Z(k)=H(k) X(k)+V(k)
\end{aligned}
$$

where

$$
\begin{aligned}
& X=\left[\Delta x^{j}, \Delta y{ }^{j}, \Delta z{ }^{j}, \Delta \dot{x}^{j}, \Delta \dot{y}{ }^{j}, \Delta \dot{z}^{j}, \Delta B^{j}, \Delta \dot{B}^{j}\right]^{T} \\
& \Phi(k)=\left[\begin{array}{cc}
\Phi_{o}(k) & 0 \\
0 & \Phi_{c}(k)
\end{array}\right] \\
& \boldsymbol{B}(\boldsymbol{k})=\left[\begin{array}{cc}
\boldsymbol{I} & 0 \\
0 & \boldsymbol{B}_{\boldsymbol{c}}
\end{array}\right] \\
& H(k)=\left[\begin{array}{ll}
H_{o}(k) & -H_{c}(k)
\end{array}\right] \\
& \boldsymbol{H}_{\boldsymbol{o}}(\boldsymbol{k})=\left[\begin{array}{cc}
l_{\underline{I}}^{\boldsymbol{j}} & 0 \\
\vdots & \vdots \\
l_{\boldsymbol{M}}^{\boldsymbol{j}} & 0
\end{array}\right] \\
& H_{c}(k)=\left[\begin{array}{cc}
1 & 0 \\
\vdots & \vdots \\
1 & 0
\end{array}\right] \\
& W(k)=\left[\begin{array}{ll}
W_{o}^{T}(k) & W_{c}^{T}(k)
\end{array}\right]^{T} \\
& V(k)=\left[\begin{array}{ll}
V_{o}^{T}(k) & V_{c}^{T}(k)
\end{array}\right]^{T}
\end{aligned}
$$

In the :above notations, we use a subscript " $\mathrm{o}$ " to represent orbit and a subscript " $\mathrm{c}$ " to represent clock. $\Phi_{\boldsymbol{O}}(\boldsymbol{k})$ characterizes the Kinematic model for ephemeris error and can be written in the foliowing formula:

$$
\Phi_{O}(k)=\left[\begin{array}{cc}
I & T I \\
0 & I
\end{array}\right]
$$

where $\mathbf{T}$ is the sampling period and $\mathbf{I}$ is an identity matrix of dimension $3 . \Phi_{c}(\boldsymbol{k})$ and $\mathbf{B}_{\mathrm{c}}$ are the midel of a sicond order Gauss-Markov process. 
Using the above model, simultaneous estimates of ephemeris and clock errors using synchronized pseudorange residuals as measurements and their sigma values to form the covariance matrix are obtained. How ever, this 4D Kalman filter approach does not successfully separate the ephemeris error from the satellite clock error [4].

\subsection{The Dynamic Algorithms}

This refers to a technique that computes precise satellite orbits from collection of measurements that are related to the satellite states by precise dynamical models accurately describing the orbit motion of the satellite. The equations describing the motion of the satellite are generally linearized with respect to a nominal trajectory for applying such technique.

Dynamic orbit determination contrasts with a Kinematic technique in which the first timederivative of the satellite position error is also estimated, again without the use of dynamics. Both the techniques can separate the ephemeris and clock errors.

When solving for GPS ephemeris errors, the state vector consists of a GPS epoch state (position and velocity), GPS clocks, station zenith tropospheric delay, WMS as well as WRS station clocks, and others biases, as well as solar pressure scaling factors for each GPS satellite. To obtain a satisfactory nominal trajectory, adequate models are to be used for gravity, third-body effect for the sun and the moon only, Solid earth tide and ocean tide and for direct solar radiation pressure[5].

\section{The satellite ephemeris error calculation}

In our simulation we suggested the ARWAAS to cover the Arab region as defined in [1] where CAIRO is selected to place master station or WMS and 7 WRS are distributed over the region. The processing algorithm is based upon a Least-Square Method for ephemeris error estimation.

\subsection{Least-Squares Algorithm}

For the satellite ephemeris determination, a least squares method is used. This method is described in the following.

In Fig. $2, \mathrm{P}_{1}$ with coordinates $\mathrm{x}_{1}, \mathrm{y}_{1}$, and $\mathrm{z}_{1}$ is the assumed position and $\mathrm{S}$ is a known position whose coordinates are $\mathrm{x}_{2}, \mathrm{y}_{2}$, and $\mathrm{z}_{2}$. The calculated distance between $\mathrm{P}_{1}$ and $S$ is then:

$$
C A=\left(\left(x_{2}-x_{1}\right)^{2}+\left(y_{2}-y_{1}\right)^{2}+\left(z_{2}-z_{1}\right)^{2}\right)^{1 / 2} \text {. }
$$

For WADGPS, two factors have to be added

1- Thes satellite clock error, $\Delta \mathrm{B}$.

2- The Master Station clock bias, $\Delta \mathrm{b}_{\mathrm{M}}$.

T"his yields:

$\mathrm{CA}=\left(\left(\mathrm{x}_{2}-\mathrm{x}_{1}\right)^{2}+\left(\mathrm{y}_{2}-\mathrm{y}_{1}\right)^{2}+\left(\mathrm{z}_{2}-\mathrm{z}_{1}\right)^{2}\right)^{1 / 2}+\mathrm{c} \cdot \Delta \mathrm{b}_{\mathrm{M}}-\mathrm{c} \cdot \Delta \mathrm{B}$. 


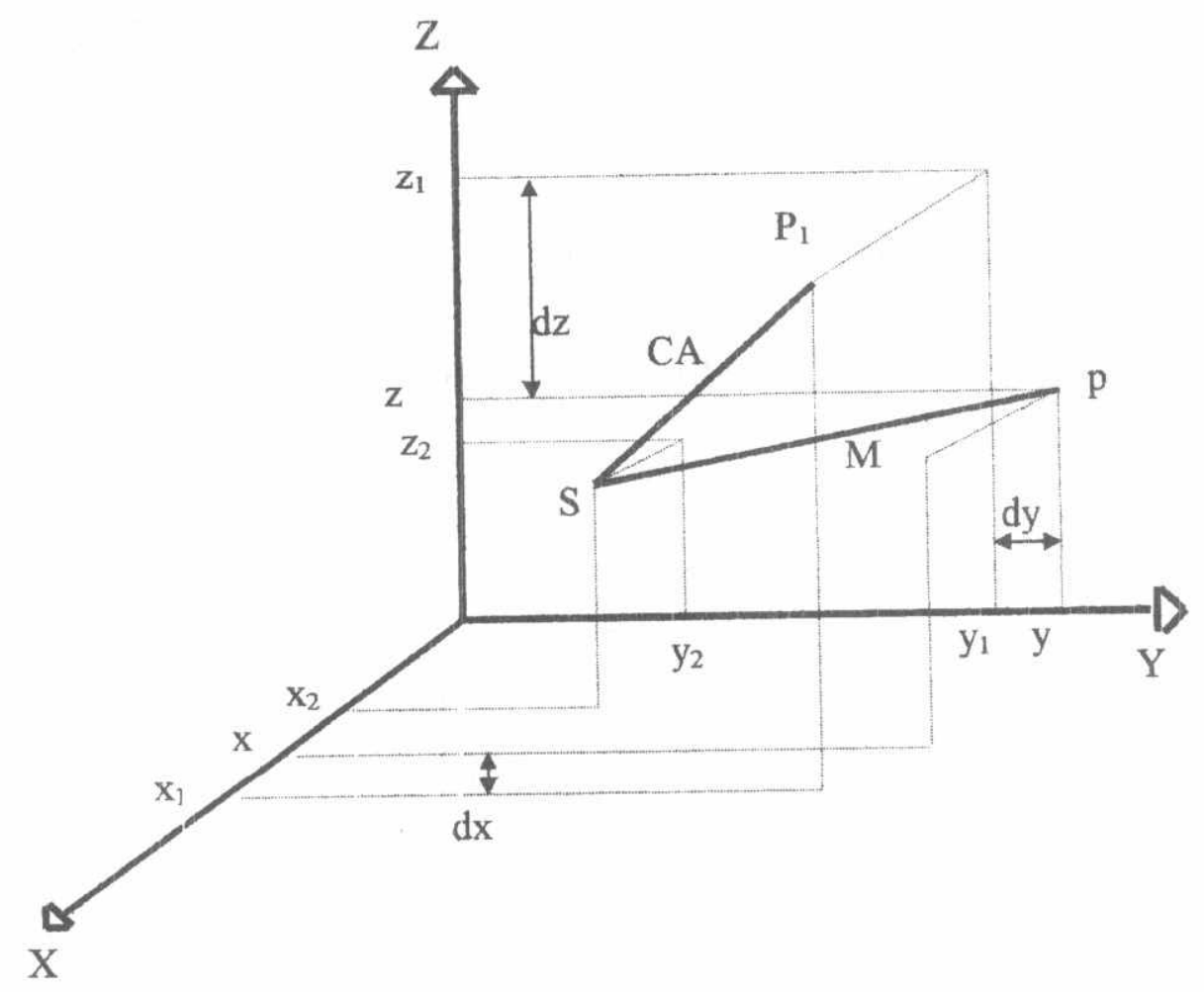

Fig(2)Distance measurement from an assumed position to a known reference.

For small change of position $\mathrm{P}_{1}$ to $\mathrm{P}$, the differential of CA is given by:

$\mathrm{dCA}=\left(\frac{-1}{\mathrm{CA}-\mathrm{c} \cdot \Delta b_{M}+c \cdot \Delta B}\right)\left[\left(\mathrm{x}_{2}-\mathrm{x}_{1}\right) \mathrm{dx}+\left(\mathrm{y}_{2}-\mathrm{y}_{1}\right) \mathrm{dy}+\left(\mathrm{z}_{2}-\mathrm{z}_{1}\right) \mathrm{dz}\right]+\mathrm{c} \cdot \mathrm{d} b_{M}-c \cdot d B$.

In other form:

$d C A=\left[\frac{x_{1}-x_{2}}{C A-c \cdot \Delta b_{M}+c . B}\right] d x+\left[\frac{y_{1}-y_{2}}{C A-c \cdot \Delta b_{M}+c \cdot B}\right] d y+\left[\frac{z_{1}-z_{2}}{C A-c \cdot \Delta b_{M}+c . B}\right] d z$

$+c \cdot d b_{M}-c \cdot d B$.

This last Equation can also be written as:

$d C A=\cos \alpha_{1} \cdot d x+\cos \alpha_{2} \cdot d y+\cos \alpha 3 \cdot d z+c \cdot d b_{M}-c \cdot d B$.

Where $\alpha_{1}, \alpha_{2}$ and $\alpha_{3}$ is the direction angle between CA and the $\mathrm{x}, \mathrm{y}$ and $\mathrm{z}$ coordinate respectively.

Let $\mathrm{k}=\cos \alpha_{1}, \quad \mathrm{l}=\cos \alpha_{2}$ and $\mathrm{u}=\cos \alpha_{3}$.

The difference between the calculated distance CA and the measured one M should be utilized to change the assumed position $\mathrm{P}_{1}$ to the (according to the measurements) correct one, $\mathrm{P}$.

The lask is then to find the corrections $d x, d y, d z, d b_{M}$ and $d B$ that reduces (M-CA) to 0 , i.e. 
However, there are five variables $d x, d y, d z, d b_{M}$ and $d B$, to be solved in $\mathrm{Eq}(7)$, so at least four more equations is needed. If more reference stations are available, we obtain equations:

$$
\begin{aligned}
& k_{1} \cdot d x+1_{1} \cdot d y+u_{1} \cdot d z+c \cdot d b_{M}-c \cdot d B=M_{1}-C A_{1} \\
& k_{2} \cdot d x+1_{2} \cdot d y+u_{2} \cdot d z+c \cdot d b_{M}-c \cdot d B=M_{2}-C A_{2} \\
& \begin{array}{l}
\cdots \\
k_{n} \cdot d x+1 \\
1_{n} \cdot d y+u_{n} \cdot d z+c \cdot d b_{M}-c \cdot d B=
\end{array}
\end{aligned}
$$

In matrix form,

$$
\text { A. } \mathrm{X}=\mathrm{B}+\mathrm{V}
$$

$$
A=\left|\begin{array}{ccccc}
k_{1} & l_{1} & u_{1} & 1 & 1 \\
k_{2} & l_{2} & u_{2} & 1 & 1 \\
k_{3} & l_{3} & u_{3} & 1 & 1 \\
\vdots & \vdots & \vdots & \vdots & \vdots \\
k_{n} & l_{n} & u_{n} & 1 & 1
\end{array}\right|, X=\left|\begin{array}{c}
d x \\
d y \\
d z \\
c . d b_{M} \\
c . d B
\end{array}\right|, B=\left|\begin{array}{c}
M_{1}-C A_{1} \\
M_{2}-C A_{2} \\
M_{3}-C A_{3} \\
\vdots \\
M_{n}-C A_{n}
\end{array}\right|, V=\left|\begin{array}{c}
v_{1} \\
v_{2} \\
v_{3} \\
\vdots \\
v_{n}
\end{array}\right|
$$

$\mathrm{V}$ is the measurement random error vector. When $\mathrm{n}>4, \mathrm{Eq}(9)$ cannot be solved exactly, but a least squares method can be used. The solution is obtained as the values of $\mathrm{dx}, \mathrm{dy}, \mathrm{dz}, \mathrm{db}_{\mathrm{M}}$ and $\mathrm{dB}$ that minimize:

$$
\sum\left(k_{i} d x+l_{i} d y+u_{i} d z+c \cdot d b_{M}-c \cdot d B-M_{i}+C A_{i}\right)^{2}
$$

Thus, the method of least squares implies that the sum of squares of the deviations is minimized. If certain measurements are assumed to be less erroneous than others, favoring weight can be put for them, giving the function:

$$
\sum w_{i}\left(k_{i} d x+l_{i} d \cdot y+u_{i} d z+c \cdot d b_{M}-c \cdot d B-M_{i}+C A_{i}\right)^{2}
$$

Where, $\quad 0<w_{i}<1$, to be minimized over $d x, d y, d z, d b_{M}$ and $d B$. Written in matrix form a minimum is sought for:

$$
F(X)=(A X-B)^{T} W(A X-B)
$$

Finding t'ne minimum of $\mathrm{F}(\mathrm{X})$ means solving the equation:

$$
\frac{d F(x)}{d X}=0
$$




$$
\begin{aligned}
\frac{d F(X)}{d X} & =\frac{d}{d X}\left[\left(\mathrm{X}^{\mathrm{T}} \mathrm{A}^{\mathrm{T}}-\mathrm{B}^{\mathrm{T}}\right)(\mathrm{WAX}-\mathrm{WB})\right] \\
& =\frac{d}{d X} \quad\left[\mathrm{X}^{\mathrm{T}} \mathrm{A}^{\mathrm{T}} \mathrm{WAX}-\mathrm{X}^{\mathrm{T}} \mathrm{A}^{\mathrm{T}} \mathrm{WB}-\mathrm{B}^{\mathrm{T}} \mathrm{WAX}+\mathrm{B}^{\mathrm{T}} \mathrm{WB}\right] \\
& =2 \mathrm{~A}^{\mathrm{T}} \mathrm{WAX}-2 \mathrm{~A}^{\mathrm{T}} \mathrm{WB}=0 .
\end{aligned}
$$

And consequently,

$$
X\left(F_{\min }\right)=\left(A^{T} W A\right)^{-1} A^{T} W B
$$

The weighting matrix is selected as the inverse of the noise covariance matrix. That is, $\mathrm{W}=\mathrm{R}^{-1}=\operatorname{cov}(\mathrm{V})$.

where $R=\varepsilon\left\{V^{T}\right\}$. This choice weights the accurate measurements more and the noisy ones less. Then the $\mathrm{Eq}(13)$ takes the form:

$$
X=\left(A^{T} R^{-1} A\right)^{-1} A^{T} R^{-1} B
$$

\subsection{Analysis of Results}

Results of the Least-Square Method for ephemeris error estimation is given for two satellite PRN 11, PRN 12 passing over the area of interest. Fig. 3 and Fiq. 4 show the estimated ephemeris error for PRN11 and PRN 12 respectively. On the same figures, the available number of stations that simultaneously view the satellite are shown. The estimated error is nearly reduced to zero for the number of WRS that see the satellite being greater than 4 .

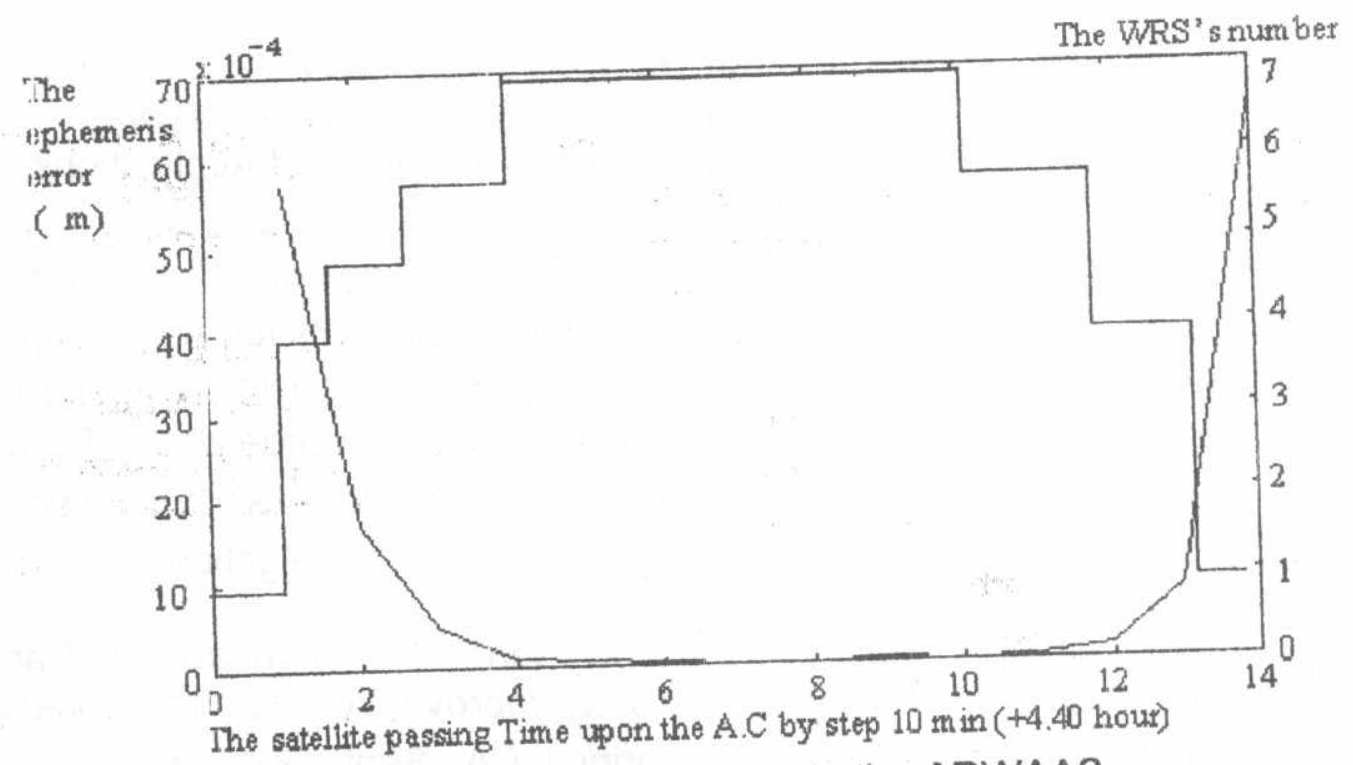

Fig(3) The PRN 11 ephemeris error in the ARWAAS. 


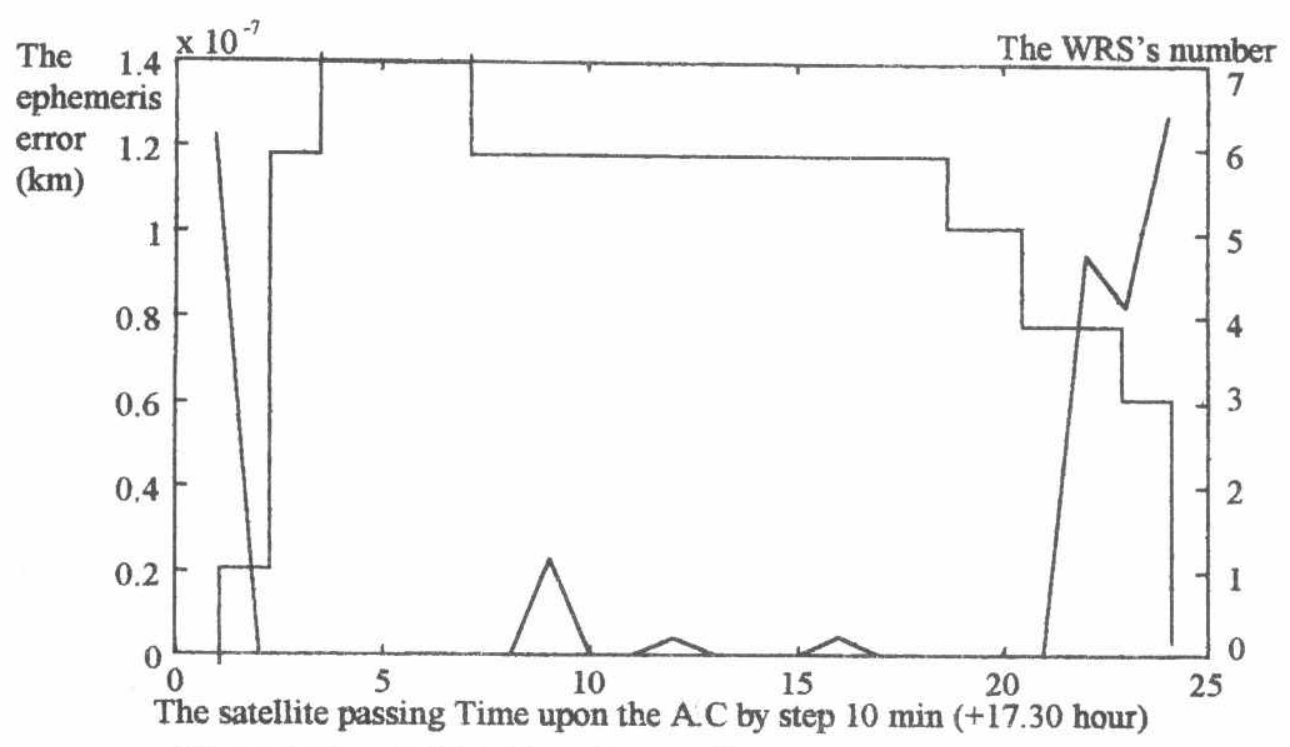

Fig(4) The PRN 12 ephemeris error in the ARWAAS.

\section{Conclusion}

Wide Area Augmentation System (WAAS) provides a vector of error corrections composed of a three-dimensional ephemeris error and clock offset for each GPS satellite plus ionospheric time delay parameters. This is done on the basis of either of the techniques used at the WMS to estimate the satellite ephemeris and clock error; namely the Nondynamic, Kinematic and Dynamic techniques.

A Least-Square Method is an effective technique for determination of the ephemeris error for the GPS satellites in the presence of relatively larger numbers of WRS monitor station to cover the required area of ARWAAS.

\section{References}

[1] S. Y. Issa, M. A. Matar, and A. Hamad, "ARWAAS Architecture and Implementation Issues", a companion paper in ASAT-8, 1999.

[2] E. D. Kaplan, 2Ed. "Understanding GPS:Principles and Applications", Chap. 7,8 published by Artech House 1996.

[3] C. Kee, T. Walter, Y. C. Chao, Y.J. Tsai, P. Enge and B. W. Parkinson," Comparison of Master Station and User Algorithms for WAAS“, Journal of Guidance, Control, and Dynamics, V 20, No 1, P.P 170-176, February 1997.

[4] Y. Tsai, Y. Chao, T. Walter, C. Kee, D. Powell, P. Enge and B. Parkinson,“ Evaluation of Orbit and Clock Models for real time WAAS“ Proceedings of the National Technical Meeting of the Institude of Navigation, Anaheim, CA, 1995.

[5] J. Ceva, W. Bertiger, R. Mullerschoen, T. Yunch and B. Parkinson, "Incorporation of Orbital Dynamics to improve WADGPS" Proceedings of ION-GPS 95, p.p. 647-659, Palm Springs, CA., Sept. 12-15, 1995. 